\title{
Hyalinizing Cholecystitis and Associated Carcinoma: A Case Report
}

\author{
Youngjin Kang · Yang-Seok Chae · Chul Hwan Kim • Youngseok Lee • Dong-Sik Kim ${ }^{1}$ Y Young-Dong Yu $•$ Joo Young Kim \\ Departments of Pathology and 'Surgery, Korea University Anam Hospital, Korea University College of Medicine, Seoul, Korea
}

Hyalinizing cholecystitis (HC) is a recently described rare variant of chronic cholecystitis that is characterized by replacement of the normal structures of the entire gallbladder wall with diffuse and dense hyaline sclerosis. ${ }^{1}$ Some cases of cholecystitis are associated with variable degrees of calcification. Porcelain gallbladder (PG) is an extensively calcific example that is a vaguely and radiologically defined entity whose pathologic correlation has not been identified. ${ }^{2-4} \mathrm{PG}$ is subclassified into "complete porcelain," showing extensive dystrophic calcifications that form an intramural continuous band involving more than $80 \%$ of the gallbladder wall, and "incomplete porcelain," which lacks these findings. ${ }^{2,5-7}$ Although $\mathrm{HC}$ is rare and found in only approximately $1.6 \%$ of cholecystectomy specimens, it is more commonly accompanied by carcinomas with more aggressive clinical behavior compared to usual gallbladder carcinomas. ${ }^{1}$ However, preoperative diagnosis of $\mathrm{HC}$-related carcinoma is challenging, because the wall is thinner than that of typical chronic cholecystitis or usual carcinomas, and discrete masses are not formed. There has been only one case report of $\mathrm{HC}$, which was accompanied by immunoglobulin G4-related disease, ${ }^{8}$ since Patel $e t a l{ }^{1}$ first described the entity in a retrospective case series. We present a case of $\mathrm{HC}$ and associated carcinoma masquerading as primary biliary cancer.

\section{CASE REPORT}

A 54-year-old man visited the hospital due to fatigue, pruritus, and diarrhea. Laboratory findings revealed elevations in aspartate

\section{Corresponding Author}

Joo Young Kim, MD, PhD

Department of Pathology, Korea University Anam Hospital, Korea University College of

Medicine, 73 Inchon-ro, Seongbuk-gu, Seoul 02841, Korea

Tel: +82-2-920-6268, Fax: +82-2-920-6576, E-mail: lepetit80@hanmail.net

Received: September 2, 2016 Revised: October 31, 2016

Accepted: November 3, 2016 aminotransferase (98 IU/L; reference range, 3 to $45 \mathrm{IU} / \mathrm{L}$ ), alanine transaminase (232 IU/L; reference range, 3 to $45 \mathrm{IU} / \mathrm{L}$ ), alkaline phosphatase (320 IU/L; reference range, 30 to $120 \mathrm{IU} / \mathrm{L}$ ), and gamma-glutamyl transpeptidase (619 IU/L; reference range, 9 to $64 \mathrm{IU} / \mathrm{L})$. Biliary computed tomography scan and magnetic resonance cholangiography revealed enhancing wall thickening of the cystic duct and common hepatic duct and multiple calcified gallstones as well as sludge in the gallbladder with mild wall thickening (Fig. 1A). Radiologically, carcinoma of the primary bile duct or cystic duct and associated chronic calculous cholecystitis was suspected, and bile duct resection with cholecystectomy was performed.

An $8.0 \times 5.5 \times 5.5 \mathrm{~cm}$ gallbladder was submitted for intraoperative frozen diagnosis. Grossly, the gallbladder wall was diffusely fibrotic, and the mucosal surface was covered by yellowish necrotic material with a gallstone $(2.0 \times 1.2 \times 1.0 \mathrm{~cm})$. There were no mass-like lesions (Fig. 1B). Two random frozen sections were examined microscopically, and an inflammatory lesion without malignancy was suggested. After the operation, we also received the segmentally resected common hepatic duct $(1.7 \mathrm{~cm}$ in length, $0.6 \mathrm{~cm}$ in diameter) and the attached cystic duct (1.7 $\mathrm{cm}$ in length, $0.4 \mathrm{~cm}$ in diameter). The bile duct wall was diffusely thickened; however, the mucosa of the ducts was smooth and did not form mass-like lesions. The entire permanent sections of the gallbladder and bile ducts were examined microscopically. The gallbladder wall was nearly completely replaced by dense lamellated eosinophilic hyaline material, with multifocal neutrophilic and lymphoplasmacytic infiltration. Focal intramural calcifications were identified (Fig. 2A, B). Multifocal invasive glands with irregular borders were sparsely scattered in the hyalinized gallbladder wall in a longitudinal arrangement (Fig. 2C). The mucosal surface of the gallbladder showed extensive denudation of the epithelium, but some foci of high-grade biliary intraepithelial neoplasia were noted (Fig. 2D). There 

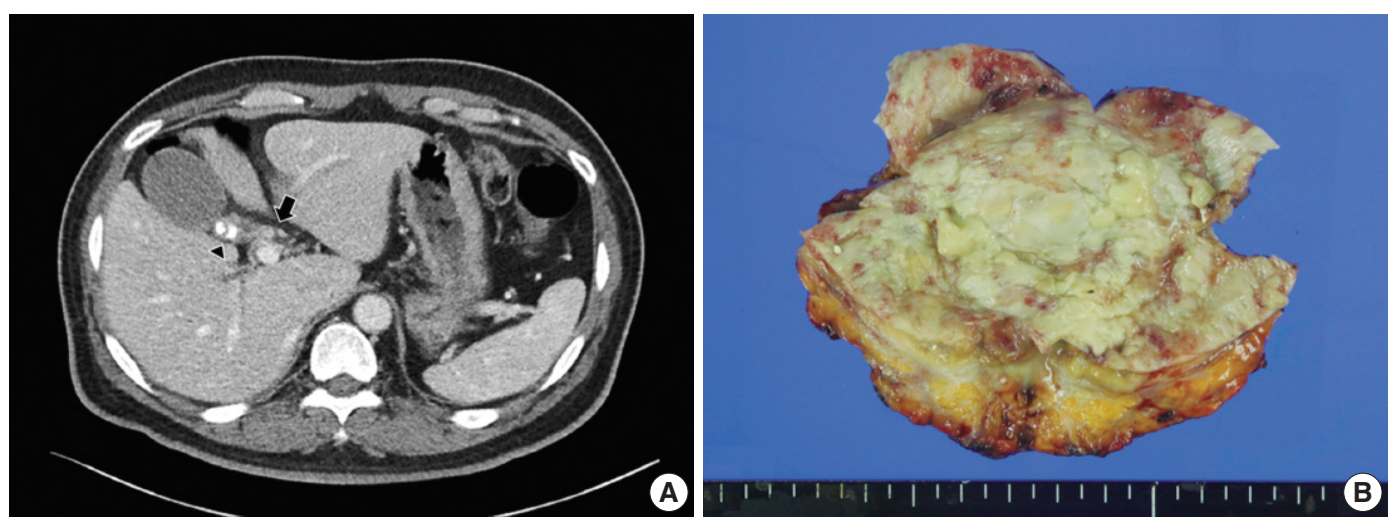

Fig. 1. Computed tomography and gross findings of hyalinizing cholecystitis and associated carcinoma. (A) Biliary computed tomography scan reveals subtle enhancement of wall thickening at the confluence of the cystic duct and the common hepatic duct (arrow). There is mild wall thickening of the gallbladder with multiple calcified gallstones in the neck portion (arrowhead). (B) Grossly, the gallbladder wall shows diffuse fibrosis and is covered by yellowish necrotic materials, without mass-like lesions.
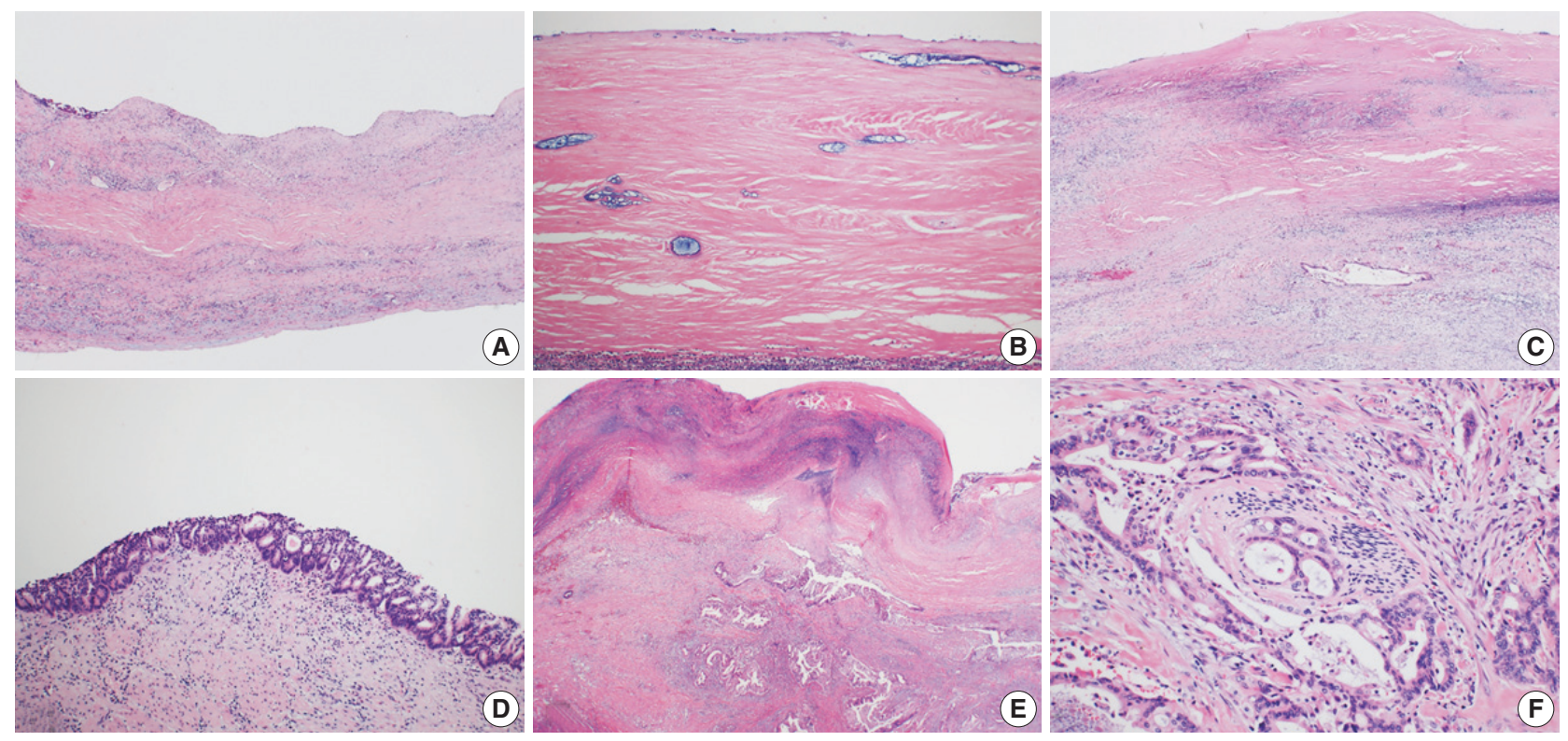

Fig. 2. Microscopic findings of hyalinizing cholecystitis and associated carcinoma. (A, B) The gallbladder wall is replaced by dense lamellated eosinophilic hyaline material. Inflammatory cells are also seen. (C) A few invasive glands are longitudinally arranged in the hyalinized galllbladder wall with a denuded epithelium. (D) Multifocal carcinoma in situ lesions are found on the surface. (E) Focal clusters of invasive glands are identified in the hyalinized wall. (F) Invasive glands have irregular borders and cytologic atypia with perineural invasion.

were focal areas composed of clusters of invasive glands in the gallbladder wall (Fig. 2E). The invasive glands were composed of stratified depolarized atypical cells with enlarged hyperchromatic nuclei containing coarse chromatin and occasionally prominent nucleoli. Some glands also showed perineural invasion (Fig. 2F). Diffuse involvement of the walls of the common hepatic duct and cystic duct by adenocarcinoma was observed (Fig. 3A, B); however, the mucosa was relatively spared, with no intraepithelial lesions (Fig. 3C). Considering all of these findings, we made the diagnosis of $\mathrm{HC}$ and associated adenocarcinoma, with direct invasion of the cystic duct and common hepatic duct.

This study was approved by the Institutional Review Board of Korea University Anam Hospital (ED17246), and informed consent was waived.

\section{DISCUSSION}

$\mathrm{HC}$ is a recently described, distinctive entity, comprising $1.6 \%$ of cholecystectomy specimens. ${ }^{1} \mathrm{HC}$ is characterized by diffuse and dense hyalinization of the gallbladder wall with 

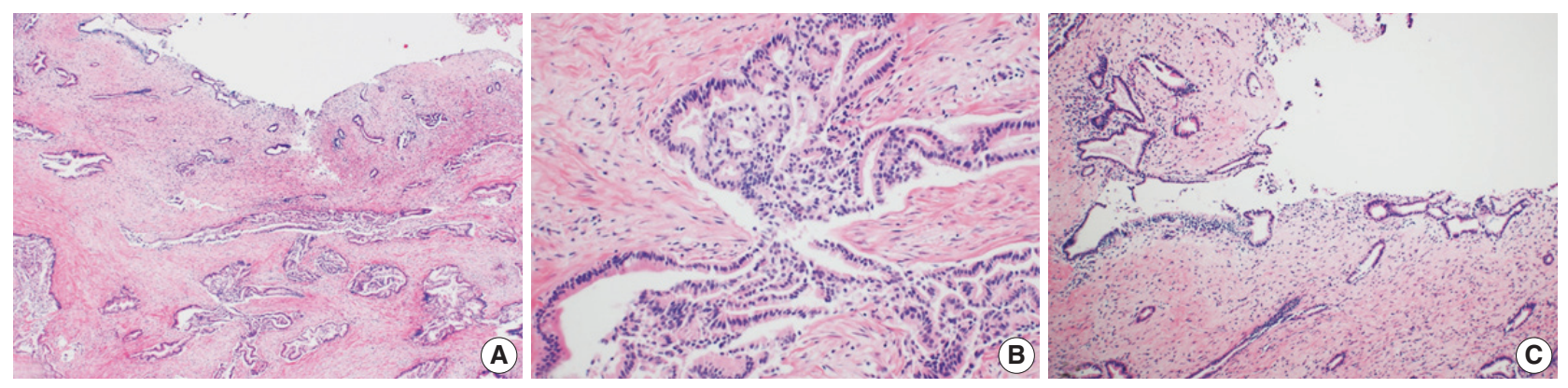

Fig. 3. Microscopic findings of the common hepatic and cystic ducts. (A, B) Invasive glands infiltrate the common hepatic and cystic ducts beneath the surface epithelium. (C) The surface epithelium is relatively spared, with no intraepithelial lesion.

complete effacement of normal histologic components. Focal to extensive calcification may also be seen. HC is more often associated with carcinoma; the frequency of carcinoma was $15 \%$ compared to $4 \%$ of all cholecystectomy specimens in a South American cohort. ${ }^{1}$ However, to identify the carcinoma component is diagnostically challenging because it does not form distinct mass lesions or induce significant thickening of the gallbladder wall. ${ }^{1}$ Furthermore, the malignant glands are not easily found due to the paucity and sparse distribution of tumor glands within an abundant hyalinizing sclerotic background. In our case, there were no mass-like lesions, and the entire gallbladder wall was diffusely fibrotic with no significant wall thickening. The carcinoma component was not found in two randomly submitted frozen sections; however, it was identified after extensive sampling of permanent sections. Therefore, careful examination with extensive or entire sampling of the gallbladder is crucial in HC to identify associated carcinoma.

Preoperative diagnosis of HC-related carcinoma is also highly challenging. Patel et al. ${ }^{1}$ reported that about $70 \%$ of malignancies had been diagnosed as benign preoperatively. This might be because HC-associated carcinomas typically do not form a mass or result in thickening of the gallbladder. Subsequently, surgical resection can be delayed until progression of the malignancy. In our case, the malignancy was identified radiologically after the tumor directly extended to the extrahepatic bile duct, and the preoperative diagnosis was primary bile duct cancer with chronic calculous cholecystitis. The final pathologic stage was T3 (direct invasion of the extrahepatic bile ducts). The late discovery of carcinoma is associated with advanced stage at diagnosis and poor prognosis of $\mathrm{HC}$-related carcinomas. The median survival for HC-associated carcinoma is shorter than that of usual carcinomas. ${ }^{1}$

In conclusion, $\mathrm{HC}$ is a rare variant of chronic cholecystitis and is often associated with carcinoma with aggressive behavior. Suspicion of the possibility of $\mathrm{HC}$ and identification of $\mathrm{HC}$ as an unusual variant of chronic cholecystitis are important in gross examination of cholecystectomy specimens. After the identification of HC, extensive sampling and meticulous microscopic examination are essential to determine the possibility of associated carcinoma. In addition, close follow-up is recommended because carcinoma associated with $\mathrm{HC}$ can have more aggressive behavior than typical gallbladder cancer.

\section{Conflicts of Interest}

No potential conflict of interest relevant to this article was reported.

\section{REFERENCES}

1. Patel S, Roa JC, Tapia O, et al. Hyalinizing cholecystitis and associated carcinomas: clinicopathologic analysis of a distinctive variant of cholecystitis with porcelain-like features and accompanying diagnostically challenging carcinomas. Am J Surg Pathol 2011;35: 1104-13.

2. Kane RA, Jacobs R, Katz J, Costello P. Porcelain gallbladder: ultrasound and CT appearance. Radiology 1984; 152: 137-41.

3. Ochsner SF, Carrera GM. Calcification of the gallbladder ("porcelain gallbladder"). Am J Roentgenol Radium Ther Nucl Med 1963; 89: 847-53.

4. Weiner PL, Lawson TL. The radiology corner: porcelain gallbladder. Am J Gastroenterol 1975; 64: 224-7.

5. Shimizu M, Miura J, Tanaka T, Itoh H, Saitoh Y. Porcelain gallbladder: relation between its type by ultrasound and incidence of cancer. J Clin Gastroenterol 1989; 11: 471-6.

6. Stephen AE, Berger DL. Carcinoma in the porcelain gallbladder: a relationship revisited. Surgery 2001; 129: 699-703.

7. Towfigh S, McFadden DW, Cortina GR, et al. Porcelain gallbladder is not associated with gallbladder carcinoma. Am Surg 2001; 67: 7-10.

8. Gupta RK, Patton KT. Hyalinizing cholecystitis with features of immunoglobulin G4-related disease-coincidence or an unrecognized association? A case report. Hum Pathol 2015; 46: 625-8. 